\title{
Comparison of Resistance and Chair Yoga Training on Subjective Sleep Quality in MCI Women
}

\author{
Maria Karydaki (Corresponding author) \\ Athens Alzheimer's Association, Athens, Greece \\ E-mail: mkarydaki@alzheimerathens.gr \\ Eleni Dimakopoulou \\ Athens Alzheimer's Association, Athens, Greece \\ E-mail: elendimakopoulou@gmail.com \\ Eleni Margioti \\ Athens Alzheimer's Association, Athens, Greece \\ E-mail: eleni_margioti@yahoo.gr \\ Vassilios Lyras \\ Athens Alzheimer's Association, Athens, Greece \\ E-mail: vassilioslyras@gmail.com \\ Xenofon Apostolopoulos \\ Athens Alzheimer's Association, Athens, Greece \\ E-mail: apostolopoulosxen@yahoo.com \\ Maria Papagianni \\ Athens Alzheimer's Association, Athens, Greece \\ E-mail: papagianni.maria@gmail.com \\ Aggeliki Vlachogianni \\ Athens Alzheimer's Association, Athens, Greece \\ E-mail: angels_vl@hotmail.com \\ Maira Theodorelou \\ Athens Alzheimer's Association, Athens, Greece \\ E-mail: mairatheodorelou@yahoo.gr \\ Paraskevi Sakka \\ Athens Alzheimer's Association, Athens, Greece \\ E-mail: vsakka@icloud.com
}

Received: 06-12- 2016

Accepted: 27-01- 2017

Published: 31-01- 2017

doi:10.7575/aiac.ijkss.v.5n.1p.26

URL: http://dx.doi.org/10.7575/aiac.ijkss.v.5n.1p.26

\begin{abstract}
Background: Self-rated sleep disorders are common in older adults, resulting in various health problems. Two types of exercise are suggested as an affordable and accessible non-pharmacological treatment and are being compared and discussed. Objectives: This randomized, controlled, 12-week trial investigates the effects of different types of exercise (resistance vs chair yoga training) on subjective sleep quality, in women with Mild Cognitive Impairment (MCI). Methods: In order to measure cognitive function, the Mini-Mental State Examination (MMSE) was used. Forty nine participants enrolled in the study were randomized to a resistance training program $(n=16)$, or a chair yoga program $(\mathrm{n}=15)$, or a control group $(\mathrm{n}=18)$. All participants engaged in cognitive activities. Results: At baseline, PSQI scores for CYG, RTG and CG $(8.2 \pm 5.1,6.1 \pm 4.3,7.4 \pm 4.1$, respectively) and MMSE $(28.3 \pm 1.4,27.8 \pm 1.2,28.0 \pm 2.3$, respectively) did not differ statistically between the three groups $\left(\mathrm{F}_{2,46}=1.993, \mathrm{p}=0.143\right)$. After the intervention, a significant
\end{abstract}


improvement in PSQI total score was noted in resistance training group $\left(\mathrm{t}=2.335, \mathrm{df}_{15}, \mathrm{p}=0.03\right)$. Conclusions: There were no significant differences between groups before and after test for the PSQI subscale scores (sleep onset latency (h), time spent in bed before sleep (min), morning waking up (h) and sleep duration (h)). No significant difference was found in PSQI subscales scores within each group. This study proposes that resistance training is an effective treatment approach to improve sleep quality in women with mild cognitive impairment.

Keywords: Exercise, chair yoga, dementia, self-rated sleep, cognitive decline

\section{Introduction}

Sleep disorders, such as trouble falling asleep and waking up repeatedly during the night, describe an under recognized although common public health problem among older adults that is stated in $50 \%$ of people aged $\geq 65$ (Foley et al., 1995), has cumulative negative impact on physical and mental health (Chaput, 2010; Kripke et al., 2002) and possibly affects quality of life (Chiba, 2013). Sleep disorders can affect daytime function in older adults and has been associated with various damaging health consequences such as anxiety, obesity, diabetes, hypertension, heart attack, stroke and an increased risk of depression (Chaput \& Tremblay, 2012; Dotto, 1996) as well as cognitive decline and functional impairment (Dotto, 1996; Bliwise, 1993; Yaffe et al., 2011). All of the above health problems along with increasing age increase the risk of Mild Cognitive Impairment (MCI). MCI is an intermediate clinical state between normal aging and dementia (Petersen et al., 1999). Older adults with MCI and dementia usually complain about insomnia or excessive daytime sleepiness (EDS) (Guarnieri \& Sorbi, 2015) that affects their overall quality of life. To date, the most common treatments for sleep problems are still pharmacological. However the size of the effect is unclear, probably due to diverse populations. Additionally the increased risk of adverse events was found to be statistically significant and potentially poses a risk factor for falls or cognitive impairment (Glass et al., 2005; Wilson et al., 2011) thus not typically recommended for long-term use (Schutte-Rodin et al., 2008). Taking into account the undesirable side effects of pharmacological interventions in addition to the large number of other drugs that older adults often take combined with insomnia medication, there is a significant need to identify safe and effective non-pharmacological approaches in order to treat sleep disorders. Previous reviews have stated that non-pharmacological treatments are as effective as pharmacological therapies for older adults with insomnia (Morin et al., 1999). Physical activity is one such alternative treatment option that is accessible, at low cost, improves mood, increase activity levels, affects numerous health systems as it has been shown that by altering certain sedentary behaviors we could modify cardiovascular risk factors (Lenz et al., 2014) and possibly improves sleep. The American Sleep Disorders Association (ASDA) states that exercise is one of the non-pharmacological interventions used to promote sleep (Hauri, 1993) and other corroborating studies have investigated the same association (Bailargeon et al., 1996; Driver and Taylor, 2000; Fetveit, 2009). It is generally believed that regular exercise could improve sleep quality and recent studies have also explored this hypothesis (Reid et al., 2010; Kamrani et al., 2014; Kashefi et al., 2014; Erlacher et al., 2015). Recent studies have demonstrated that, yoga training have improved self-reported sleep quality in addition to improved quality of life and decreased depression in older adults (Hariprasad et al., 2013). In addition, aerobic and anaerobic simple exercises have shown a significant effect on depressive symptoms of women with renal transplants (Shakoor et al., 2016). Likewise, moderate-intensity exercise such as low impact aerobics, brisk walking and stationary cycling can be beneficial in healthy sedentary older the effects of various types of exercise on sleep in older adults with MCI are still limited and the evidence for a positive effect on sleep is sparse with unclear findings mainly due to the limited number of participants. Most studies examining the effects of exercise on sleep have focused on moderate aerobic exercise (Sharif et al., 2015; Lira et al., 2011). In addition, to the best of our knowledge, there is limited data available in chair yoga intervention and in resistance exercise (Faria et al., 2009; Ferris et al., 2005) evaluating the effects of these types of exercise on sleep quality.

\section{Methods}

\subsection{Study Design}

This is a randomized controlled study approved by the Ethical Committee of the Greek Alzheimer Association and met the ethical standards (Harriss \& Atkinson, 2015). Subjects willing to participate in the study signed a written consent after oral information about the objectives of the study. Each Day Care Center of the Athens Alzheimer Association and Related Disorders as a block was randomized to chair yoga (CYG), resistance training (RTG) or control group (CG) by an independent researcher who was not involved in assessments (Figure 1). 


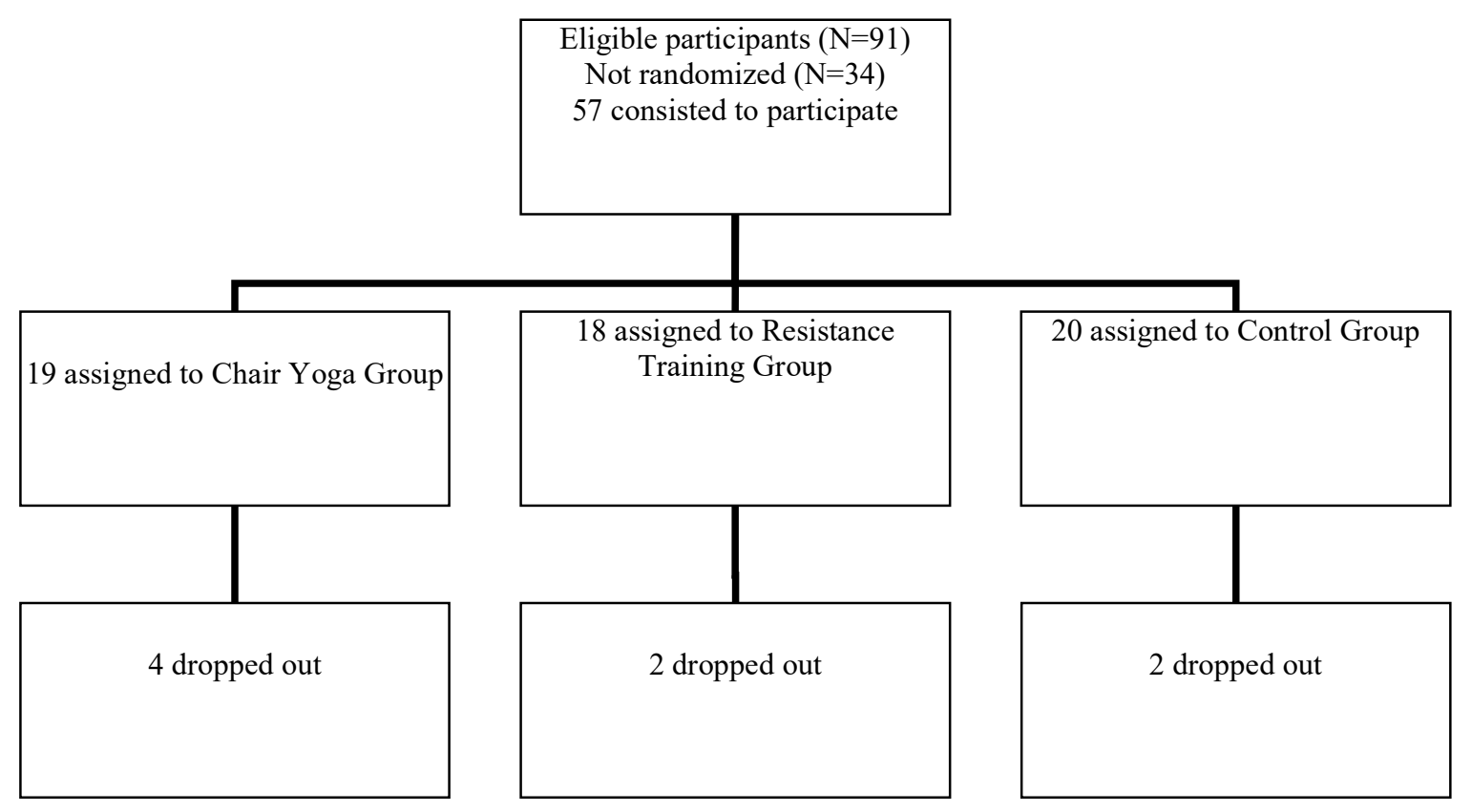

Figure 1. Flow chart demonstrating random allocation of the subjects to the three groups

\subsection{Participant Characteristics}

Participants $(n=49)$ included in the study fulfilled diagnostic criteria (Petersen \& Morris, 2005). To assess cognitive function the Mini-Mental State Examination (MMSE) was used. The participants should not have been engaged in any other type of physical activity over the previous 3 months. Other excluding factors were major depressive disorder and an inability to perform a physical exercise program due to medical reasons, namely clinical evidence of unstable cardiac disease, uncontrolled hypertension and pulmonary disease such as asthma. The reasons for the 34 not randomized in the study were due to lack of interest $(\mathrm{N}=16)$, refusal to participate $(\mathrm{N}=18)$. From the forty nine $(\mathrm{N}=49)$ participants enrolled in the study, 16 assigned to resistance training group (RTC), 15 to chair yoga group (CYG) and 18 to control group (CG). All participants engaged in cognitive activities.

\subsection{Materials and Procedures}

Body weight $(\mathrm{kg})$ and height (meters) were measured in order to determine BMI $\left(\mathrm{kg} / \mathrm{m}^{2}\right)$. Weight was measured to the nearest $0.1 \mathrm{~kg}$ on a portable digital body scale and height was measured to the nearest $0.5 \mathrm{~cm}$ on a stable stadiometer. All participants completed the Senior Fitness Test in order to assess their physical fitness level (Rikli \& Jones, 1999).This battery measures a variety of physiological parameters and functional activities and is especially useful for tracking a person over time It is an assessment tool developed and validated in older adults, quick and easy to administer and has comprehensible instructions for the participants. It is low-cost and requires easy-to-use equipment. All groups were tested before intervention and after 12 weeks intervention period. On the test day, participants firstly performed a 10 minute warm up under the guidance of an exercise instructor and then completed the senior fitness test items. Warm up included head half circles and turns, single arm crossovers, check stretch, calf and hamstringing stretch (Rikli \& Jones, 2001). Moreover, participants completed three days before assessment, one trial of each test item to become familiarized with the assessment test items, according to the recommendations of Rikli \& Jones (1999). Scoring was then noted on a performance sheet. Senior fitness test is a widely used standardized and safe assessment for providing information about physical fitness in older people with mild cognitive impairment (Hesseberg et al., 2016; Lee SH et al., 2016). Six assessment items are involved in the Senior fitness test (Rikli \& Jones, 2001) and will be described briefly: 1) arm curl for upper body strength. The score is the total number of hand-weight curls performed in 30sec, 2) 30-Second Chair Stand to assess lower body strength and the score is the total number of stands executed within 30sec, 3) 2-Minute Step Test (aerobic endurance). The score is the number of times right knee reaches the required height, 4) Chair Sit-and-Reach (lower body flexibility) and the score is the distance achieved between extended fingers and tip of toe, 5), Back Scratch measures the upper body flexibility and the score is the distance between extended middle fingers, and 6) 8-foot Up-and-Go to measure agility and dynamic balance and the score is the best time achieved to get up from a seated position, walk $2.44 \mathrm{~m}$, turn, and return to seated position. The test battery was performed before and after the 12-week intervention under the same conditions. Berg Balance Scale (Berg et al., 1992) is widely used 14-item scale designed to measure and assess the balance of the older adults using a performance score. Subjective sleep quality was measured by the validated self-rated sleep questionnaire PSQI at the baseline and after 12 weeks. There are 18 individual items, with the majority of the questions utilizing a 4-point Likert-type scale, that generate seven "component" scores, each with a score ranging from 0 (no difficulty) to 3 (severe difficulty). The seven component scores are namely: Subjective sleep quality, sleep latency, sleep duration, habitual sleep efficiency, sleep 
disturbances, medication use and daytime dysfunction due to poor sleep quality.

\subsection{Intervention}

The 45-minute classes, twice per week were guided by certified fitness instructors. Classes, of chair yoga and resistance training, were held at the Day Care Centers of Alzheimer Association under the same conditions.

\subsection{Resistance Training Program}

A resistance training class was 45-minute long, twice per week, and consisted of a 10-minute warm-up, 30 minutes of basic content and a 5-minutes cool-down. Free weights, elastic bands, medicine balls and pilates rings were used as training stimulus with free weights ranging from 1 to $1.5 \mathrm{~kg}$. The basic key resistance exercises are presented in Table 1. A $30 \mathrm{sec}$ rest interval was used between sets.

Table 1. Resistance training programme (12 week duration, twice/week)

\begin{tabular}{|c|c|c|c|c|}
\hline Exercise & 1-3 week & 3-6 week & 6-9 week & 9-12 week \\
\hline & Volume (set $\mathrm{x}$ rep) & & & \\
\hline Leg squats & $2 \times 8$ & $2 \times 10$ & $2 \times 14$ & $3 \times 12$ \\
\hline Toe stands & $2 \times 12$ & $2 \times 15$ & $2 \times 18$ & $3 \times 15$ \\
\hline Side hip raise & $2 \times 10$ & $2 \times 12$ & $2 \times 15$ & $3 \times 12$ \\
\hline $\begin{array}{l}\text { Hip adductions } \\
\text { (pilates ring) }\end{array}$ & $2 \times 15$ & $2 \times 18$ & $2 \times 22$ & $2 \times 20$ \\
\hline Biceps curls & 2x8 (1kg weight) & $2 \times 10$ (1kg weight) & $\begin{array}{l}2 \times 12(1.5 \mathrm{~kg} \\
\text { weight })\end{array}$ & $\begin{array}{l}2 \times 15(1.5 \mathrm{~kg} \\
\text { weight) }\end{array}$ \\
\hline Triceps extensions & 2x8 (1 kg weight) & $2 \times 10$ (1 kg weight) & $\begin{array}{l}2 \times 12(1.5 \mathrm{~kg} \\
\text { weight })\end{array}$ & $\begin{array}{l}2 \times 15(1.5 \mathrm{~kg} \\
\text { weight })\end{array}$ \\
\hline $\begin{array}{l}\text { Shoulder press } \\
\text { ( } 2 \mathrm{~kg} \text { medicine } \\
\text { ball) }\end{array}$ & $2 \times 8$ & $2 \times 10$ & $2 \times 15$ & $3 \times 10$ \\
\hline $\begin{array}{l}\text { Resistance band } \\
\text { row }\end{array}$ & $2 \times 10$ & $2 \times 12$ & $2 \times 15$ & $3 \times 12$ \\
\hline plank & $2 \times 10 \mathrm{sec}$ & $2 \times 15 \mathrm{sec}$ & $2 \times 20 \mathrm{sec}$ & $2 \times 30 \mathrm{sec}$ \\
\hline
\end{tabular}

\subsection{Chair Yoga}

The chair yoga program was created by combining traditional and contemporary yoga and chair yoga practices. It was then adapted in order to be appropriate for older adults with MCI. The chair yoga program group included of loosening exercises, physical postures, and breathing exercises. More specifically the loosening exercises included of exercises correcting the alignment of the body, forward bends, backbends, side stretches and some twists using the chair. The physical postures comprised of standing poses (promoting strength, flexibility, balance, stability and spatial orientation), and relaxation poses. Breathing exercises were an integral part of each session (all participants went through a breathing assessment in order to determine whether they are normal or reverse breathers), as it accompanies every yoga pose. No inversions were executed. Participants attended the classes 2 days/week. Details of chair yoga programe is showed in Table 2. The rest interval between exercises was one minute. 
Table 2. Chair yoga programme (12 weeks duration, twice/week)

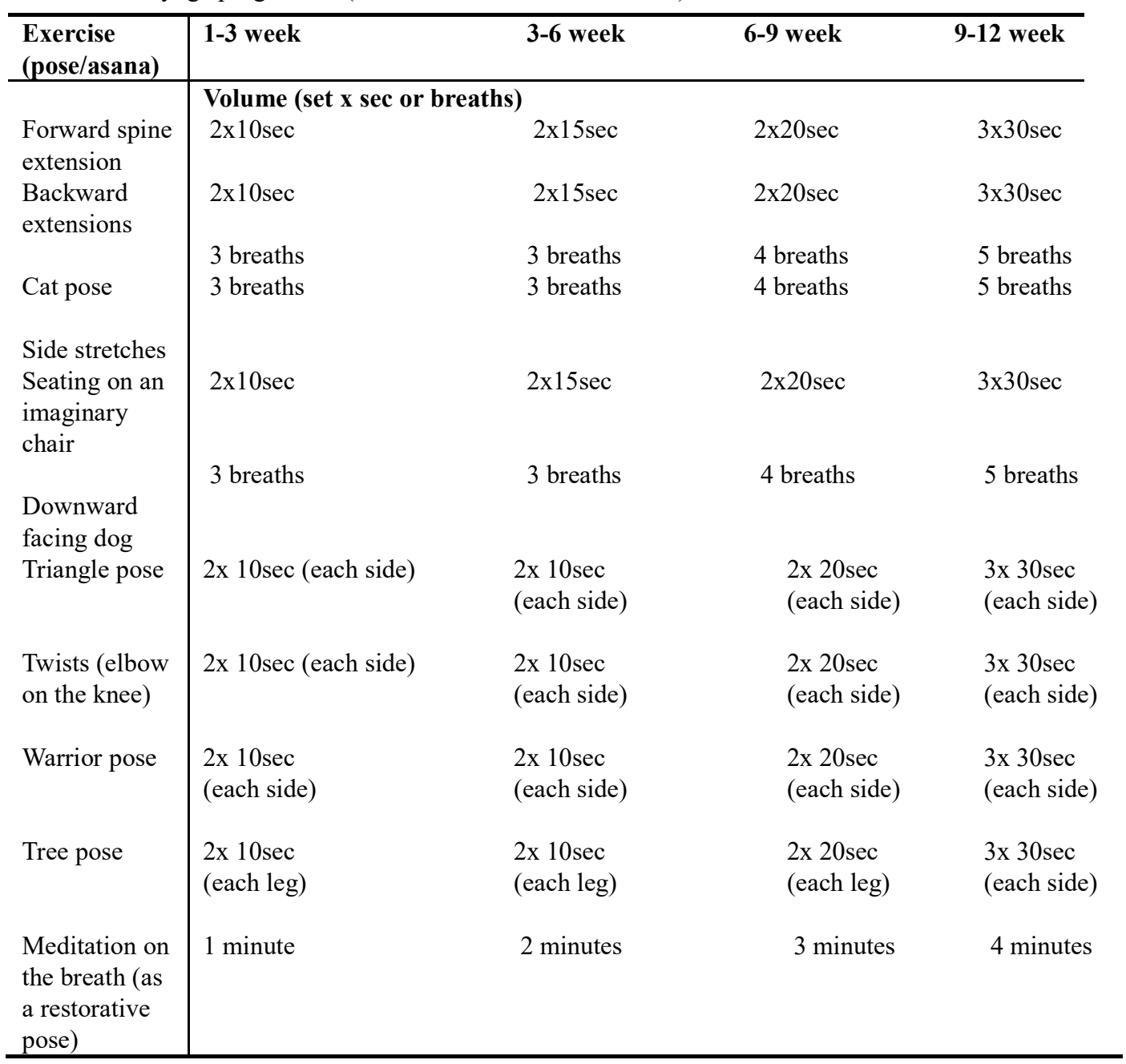

\subsection{Control Group}

All the participants of control group were asked to refrain from any complementary exercise during the course of the study (12 weeks). The 18 participants in the control group engaged in cognitive activities twice a week 2 hours a day.

\subsection{Statistical Analysis}

The Kolmogorov- Smirnov (KS-test) was applied to test the normal distribution of variables. Paired t-test was implemented to investigate the effect of the intervention within groups. To investigate the differences among the three groups a one-way analysis of variance (ANOVA repeated) was performed and the pair-wise comparisons were adjusted using the Holm-Sidac procedure. The non-parametric Kruskal-Wallis ANOVA on ranks was employed in order to test for differences among the groups if the data did not follow a normal distribution. The Pearson t-product was used to explore correlations. All results are presented as Mean \pm SD. The statistical analysis was conducted using SPSS IBM 22. Statistical significance was set at $<0.05$.

\section{Results}

The total number of participants were 49 women (72.6 $\pm 5.9 \mathrm{yrs})$ assigned to three groups (CYG (N=15), RTG (N=16), CG (N=18)). There were no differences in MMSE score between CYG (28.3 \pm 1.4$)$, RTG (27.8 \pm 1.2$)$ and CG (28.0 \pm 2.3$)$. A significant difference was noted in age $(y r s)$ between groups $\left(F_{2,46}=14.210, p=0.001\right)$ and more specifically, the participants in CYG $(67.2 \pm 4.54 \mathrm{yrs})$ were younger than participants in the RTG $(75.6 \pm 4.08 \mathrm{yrs})$ and the CG (74.51 $\pm 5.46 \mathrm{yrs})$. There were no statistically significant differences (Table 3) between and within groups for weight (kg) and BMI $\left(\mathrm{kg} / \mathrm{m}^{2}\right)$.

Table 3. Participant physical characteristics before and after the interventions

\begin{tabular}{l|llllll}
\hline Variables & \multicolumn{2}{l}{ CYG $(n=15)$} & \multicolumn{3}{l}{ RTG $(n=16)$} & \multicolumn{3}{l}{$C G(n=18)$} \\
\hline & Before & After & Before & After & Before & After \\
Height $(\mathrm{cm})$ & $157.0 \pm 01$ & $157.0 \pm 0.1$ & $156.6 \pm 0.1$ & $156.5 \pm 0.2$ & $159.3 \pm 0.3$ & $158.9 \pm 0.2$ \\
Weight, $(\mathrm{kg})$ & $74.2 \pm 12.2$ & $74.2 \pm 12.3$ & $70.3 \pm 14.5$ & $70.5 \pm 14.4$ & $72.1 \pm 9.5$ & $71.6 \pm 8.8$ \\
\hline BMI $\left(\mathrm{kg} / \mathrm{m}^{2}\right)$ & $30.02 \pm 3.91$ & $30.04 \pm 3.91$ & $28.5 \pm 4.1$ & $28.5 \pm 4.0$ & $28.3 \pm 2.43$ & $28.2 \pm 2.17$ \\
\hline
\end{tabular}


At baseline, PSQI total score did not differ statistically between CYG, RTC and CG $\left(\mathrm{F}_{2,46}=1.993, \mathrm{p}=0.143\right)$. After the intervention (Table 4), no significant difference was found between groups in PSQI total score $\left(\mathrm{F}_{2,45}=0.028, \mathrm{p}=0.972\right)$. Further analysis, indicated that no improvement was noted in subjective sleep quality within groups, with the exception of RTG $\left(\mathrm{t}=2.335, \mathrm{df}_{15}, \mathrm{p}=0.03\right)$. There were no significant differences between groups before and after test for the PSQI subscale scores (sleep onset latency (h), time spent in bed before sleep ( $\mathrm{min}$ ), morning waking up (h) and sleep duration (h)). No significant difference was found in PSQI subscales scores within each group (Table 4).

Table 4. PSQI total score and subscales of each group before and after the intervention (Mean \pm SD)

\begin{tabular}{|c|c|c|c|c|c|c|}
\hline Variables & $C Y G(n=15)$ & $R T G(n=16)$ & & $C G(n=18)$ & & \\
\hline & Before & After & Before & After & Before & After \\
\hline PSQI score & $8.2 \pm 5.1$ & $7.1 \pm 3.9$ & $6.1 \pm 4.3^{*}$ & $5.1 \pm 3.4$ & $7.4 \pm 4.1$ & $7.0 \pm 2.8$ \\
\hline $\begin{array}{l}\text { Sleep onset } \\
\text { latency }(\mathrm{h})\end{array}$ & $23.8 \pm 0.68$ & $23.6 \pm 0.62$ & $23.3 \pm 0.9$ & $23.5 \pm 0.87$ & $22.7 \pm 0.87$ & $22.7 \pm 0.87$ \\
\hline $\begin{array}{l}\text { Time spent in } \\
\text { bed before } \\
\text { asleep (min) }\end{array}$ & $38.3 \pm 25.03$ & $53.3 \pm 36.60$ & $40.0 \pm 43.9$ & $25.0 \pm 20.1$ & $30.0 \pm 9.48$ & $25.8 \pm 14.9$ \\
\hline $\begin{array}{l}\text { Morning } \\
\text { waking up (h) }\end{array}$ & $7.38 \pm 0.87$ & $7.6 \pm 1.06$ & $7.8 \pm 0.7$ & $7.8 \pm 0.9$ & $7.4 \pm 0.8$ & $7.5 \pm 1.03$ \\
\hline $\begin{array}{l}\text { Sleep duration } \\
\text { (h) }\end{array}$ & $6.3 \pm 1.06$ & $6.6 \pm 1.03$ & $6.5 \pm 1.64$ & $6.5 \pm 1.64$ & $5.6 \pm 1.03$ & $6.3 \pm 1.50$ \\
\hline
\end{tabular}

\subsection{Changes in Fitness test}

At baseline, no significant differences were noted between groups in the functional fitness test. The CYG and RTG had differences as well as the CG in arm curl and 30sec- chair stand test $(p=0.001)$. After the 3 months intervention program the RTG showed an improvement in up \& go test (from 5.8 to $5.1 \mathrm{sec}, \mathrm{p}=0.001$ ) and a small decrease in 30secchair stand performance (from 16 to $14 \mathrm{rep}, \mathrm{p}=0.002$ ). For women in the $\mathrm{CYG}$ a significant increase in 2-Minute step test was found (from 72 to 79 steps, $\mathrm{p}=0.023$ ) and a decrease in arm curl test (from 23 to 22 rep., $\mathrm{p}=0.023$ ). We also found that women in CYG, improved their balance (from 55 to 56 score, $\mathrm{p}=0.022$ ). No improvements were found in the CG in any of the functional fitness test items after the intervention. Additionally, after the 12-week intervention, differences were found between the three groups. More specifically, the RTG and CYG, differed in 30sec- chair stand test from CG $(\mathrm{p}=0.001)$. Participants of RTG demonstrated a better performance in up \& go test compared to CG $(\mathrm{p}=0.001)$. Likewise, women of CYG scored better in up \& go test, in comparison to CG (6.24 vs $8.04 \mathrm{sec}, \mathrm{p}=0.001)$.

\section{Discussion}

A 12-week exercise intervention was linked to self-reported sleep quality in a sample of 49 women diagnosed with MCI. Their physical fitness level was measured using The Senior fitness test and Berg Balance Scale, while self-rated sleep quality was evaluated using The Pittsburg Sleep Quality Index (PSQI). Surprisingly, a significant increase in 2Minute step test, an item measuring stamina, was noted in the CYG albeit it is an anaerobic form of exercise mainly aiming at improving balance, posture and flexibility. This result is similar to a study that found an improvement in cardiopulmonary endurance after a yoga exercise intervention (Tran et al., 2001, Ramos-Jimenez et al.,2009; Balasubramanian \& Pansare, 1991). According to our results, a possible explanation for the increase in endurance (2 Minute step test) was related to previous low aerobic fitness of our sample. It seems that yoga training stimulus is enough to improve or maintain cardiovascular fitness (Kharti et al., 2007; Innes \& Vincent, 2007; Yang, 2007). As expected, an improvement in balance was noted in the CYG participants. However, the results in arm curl test, which measures the upper body strength, in the same group showed a slight decrease. A possible reason for this slight decrease, is that in our study most chair yoga exercises derived from holding static postures, and so static or isometric contractions do not reliably lead to increases in isokinetic strength (Lindh, 1979). In the RTG the up \& go test showed an improvement while a small decrease was noted in $30 \mathrm{sec}$ chair stand performance. These results regarding the two intervention groups suggest that fitness levels are possibly influenced by various factors (i.e. previous activity levels and current health status). Regarding subjective sleep quality, an improvement was noted in the resistance training group. Consistent with this study's finding mentioned above, one study presented that progressive resistance training improved all aspects of subjective sleep quality (tBrockton, 1997). Additionally, a recent pilot study showed a $38 \%$ improvement in subjective sleep quality of sedentary older adults, after a six-month long resistance training program (Ferris et al., 2005). However, in the current study, there were no significant pre and post-test differences between groups or within each group in PSQI subscale scores, nonetheless, the intervention groups yielded a better global PSQI score, compared to the control group. It is likely that the frequency of the intervention (2days/week) may not be sufficient. Existing studies with positive results included greater-frequency exercise intervention (Baker et al., 2010, Carral \& Perez, 2007). The optimal dose of physical activity presently remains unclear and needs investigation. 
Moreover, a longer intervention period ( $>12$ weeks) might have produced sleep improvements, based on the assumption that this period of time would be long enough to detect improved sleep scores. Although one study with a 6 months tai chi exercise intervention observed significant improvements in five of the PSQI subscale scores compared to the lowimpact exercise participants, however, the study group consisted of a very wide age range (60-92 years old) with no cognitive deficits (Li et al., 2004). Li et al. (2004) findings suggest tai chi as more effective than any other low-intensity exercise.

In another RCT conducted in older adults reporting cognitive and sleep complaints, the intervention was various types of physical and mental activity for 12 weeks, and the authors concluded that a low-intensity exercise combined with mental activities improved self-reported sleep quality (Pa et al., 2014). Overall, benefits on various sleep parameters may be influenced by study participants, since their baseline characteristics in other studies varied in physical activity levels or the level of cognitive problems. This study agrees with the findings of a number of studies that investigated different types of physical activity in various frequencies. An RCT conducted by King et al. (1997) to explore the effects of a low-impact aerobic exercise training on self-rated sleep quality among healthy, older adults with sleep complaints, revealed significant improvement in the PSQI global score after 16 weeks. One RCT similar to the current study explored the influence of a tai chi program in 65 older adults residing in an elderly home, 3 times/week over a 12 weeks period, showed a positive, significant effect on their sleep quality (Hosseini et al., 2011). The influence of chair yoga on sleep quality has not been given enough attention. In this study women of CYG scored better in up \& go test, in comparison to CG (6.24 vs $8.04 \mathrm{sec}, \mathrm{p}=0.001)$.

Previous studies have discussed the role of chair yoga or yoga adapted to the needs of older adults, with positive results, but this type of exercise needs further investigation. In a recent study by Oken, et al., 2006) of one hundred thirty-five healthy individuals aged 65-85 years were recruited and randomized into three groups (Hatha yoga, walking, and control). Participants in the yoga group displayed significant improvement in quality of life and some physical measures compared to walking and control group. In another recent study of older adults with mild to severe AD, a 10-week chair yoga intervention showed a significant effect in ADLs, however no significant change in balance, anxiety, or cognition was noted (Litchkey et al., 2012). It is probable that with a longer intervention and a bigger sample size, yoga could possibly show a bigger effect and suggest an important role in the improvement in the quality of life in people with AD.

\subsection{Strengths}

The key strength of this study is that it was an RCT, generally considered as the gold standard, aimed to investigate and associate the effects of various types of exercise on sleep quality, using women with MCI as a sample. The majority of studies reported the impact of exercise on sleep in healthy participants or the effect of exercise on cognitive symptoms in older people with dementia, without measuring their sleep quality as well. The present study was specifically designed in order to associate the effects of two different types of exercise on sleep quality in women with MCI. The exercise programs described in this study are using equipment easily accessible and inexpensive, so the majority of people can do them, with the guidance of a certified fitness instructor.

\subsection{Limitations}

PSQI is a subjective measure of sleep quality. Additional studies are required in order to support these findings using objective sleep quality measures (i.e. polysomnography). Moreover, 12 weeks was too short time to reflect changes related to either type of exercise. Although a significant improvement was noted in various sleep parameters over 12 weeks, it would be interesting to investigate the effects of exercise over a longer period, i.e. 16 weeks. All exercise classes were led by a qualified exercise instructor, making an unsupervised activity at home inappropriate and possibly harmful. Finally, the study population consisted only of women with MCI, so these findings cannot be generalized to men, although the MCI population is a major target group for physical and mental activity interventions that could possibly promote a range of health-enhancing effects. Older adults with cognitive complaints, who are already participating in some form of systematic physical activity or with more severe sleep problems that require medical treatment, might not experience the same positive effects.

\section{Conclusion}

The current study's results suggest that resistance exercise programs may offer an effective, inexpensive method in order to improve sleep quality in older adults with MCI and subjective sleep problems. Assuming that lack of sleep and MCI are identified as risk factors for cognitive decline and dementia, it is probable that by improving sleep quality through a combination of simple exercise programs could alleviate future cognitive decline. More studies with a bigger sample size and a longer intervention are needed in order to define long term or maintenance effects of different types of exercise on sleep and cognition.

\section{References}

Baillargeon, L. U. C. I. E., Demers, M., Gregoire, J. P., Pepin, M. (1996). Study on insomnia treatment by family physicians. Canadian family physician Medecin de famille canadien, 42, 426-432.

Baker, L.D., Frank, L.L., Foster-Schubert, K., et al. (2010). Effects of aerobic exercise on mild cognitive impairment: a controlled trial. Archives of neurology, 67(1), 71-79.

Balasubramanian, B., Pansare, M.S. (1991). Effect of yoga on aerobic and anaerobic power of muscles. Indian Journal Physiology Pharmacologica, 35(4), 281-282. 
Berg, K.O., Wood-Dauphinee, S.L., Williams, J.I., Maki, B. (1992). Measuring balance in the elderly: validation of an instrument. Canadian Journal of Public Health, 83 Suppl 2, S7-11.

Bliwise, D.L. (1993). Sleep in normal aging and dementia. Sleep: Journal of Sleep Research \& Sleep Medicine, 16(1), 40-81.

Carral, C.J.M., Perez, C.A. (2007). Effects of high-intensity combined training on women over 65.Gerontology, 53(6), 340-346.

Chaput, J.P. (2010). A good night's sleep for a healthier population. American journal of preventive medicine, 38(3), 349.

Chaput, J.P., Tremblay, A. (2012). Insufficient sleep as a contributor to weight gain: an update. Current obesity reports, 1(4), 245-256.

Chiba, S. (2013). Sleep disorders in older adults. Nihon rinsho. Japanese journal of clinical medicine, 71(10), 17631774 .

Dotto, L. (1996). Sleep stages, memory and learning. CMAJ: Canadian Medical Association Journal, 154(8), 1193.

Driver, H. S., Taylor, S. R. (2000). Exercise and sleep. Sleep medicine reviews, 4(4), 387-402.

Elacher, C., Elacher, D., Schredl, M. (2015). The effects of exercise on self-rated sleep among adults with chronic sleep complaints. Journal of Sport and Health Science, 4, 289-298.

Faria, A.P., Cavagnolli, D.A., Rossi, M.V., et al. (2009). Effects of resistance exercise on the sleep patterns of sedentary individuals. Sleep science, 2(3), 141-146.

Ferris, L. T., Williams, J. S., Shen, C. L., et al. (2005). Resistance training improves sleep quality in older adults-a pilot study. Journal of Sports Science and Medicine, 4(3), 354-60.

Fetveit, A. (2009). Late-life insomnia: A review. Geriatrics \& Gerontology International, 9(3), 220-234.

Foley, D.J., Monjan, A.A., Brown, S.L., Simonsick, E.M. (1995). Sleep complaints among elderly persons: an epidemiologic study of three communities. Sleep: Journal of Sleep Research \& Sleep Medicine, 18(6), 425-432.

Glass, J., Lanctôt, K.L., Herrmann, N., et al. (2005). Sedative hypnotics in older people with insomnia: meta-analysis of risks and benefits. British Medical Journal, 331(7526), 1169.

Guarnieri, B., Sorbi, S. (2015). Sleep and Cognitive Decline: A Strong Bidirectional Relationship. It Is Time for Specific Recommendations on Routine Assessment and the Management of Sleep Disorders in Patients with Mild Cognitive Impairment and Dementia. European neurology, 74(1-2), 43-48.

Hariprasad, V.R., Sivakumar, P.T., Koparde, V., et al. (2013). Effects of yoga intervention on sleep and quality-of-life in elderly: A randomized controlled trial. Indian journal of psychiatry, 55(Suppl 3), S364.

Harriss, D.J., Atkinson, G., (2015). Ethical standards in sport and exercise science research: 2016 update. International Journal of Sports Medicine, 36, 1121-1124.

Hauri, P.J. (1993). Consulting about insomnia: a method and some preliminary data. Sleep: Journal of Sleep Research \& Sleep Medicine, 16(4), 344-50.

Hesseberg, K., Bergland, A., Rydwik, E., Brovold, T. (2016) Physical Fitness in Older People Recently Diagnosed with Cognitive Impairment Compared to Older People Recently Discharged from Hospital. Dementia Geriatric Cognitive Disorders Extra, 6, 396-406.

Hosseini, H., Esfirizi, M.F., Marandi, S.M., Rezaie, A. (2011). The effect of Tai Chi exercise on the sleep quality of the elderly residents in Isfahan, Sadeghieh elderly home. Iranian journal of nursing and midwifery research, 16(1).

Innes, K.E., Vincent, H.K., (2007). The influence of yoga-based programs on risk profiles in adults with type 2 diabetes mellitus: A systematic review. Evidenced Based Complement Alternative Medicine, 4, 469-86.

Kamrani, A.A.A., Shams, A., Dehkordi, P.S., Mohajeri, R. (2014). The effect of low and moderate intensity aerobic exercises on sleep quality in elderly adult males. Pakistan Journal of Medicine Science, 30(2), 417-421.

Kashefi, Z., Mirzaei, B., Shabani, R. (2014). The Effects of Eight Weeks Selected Aerobic Exercises on Sleep Quality of Middle-Aged Non Athletes Females. Iran Red Crescent Medicine, 16(7), e16408.

Khatri, D., Mathur, K.C., Gahlot, S., et al. (2007). Effects of yoga and meditation on clinical and biochemical parameters of metabolic syndrome. Diabetes Research Clinical Practice, 78,e9-10.

King, A.C., Oman, R.F., Brassington, G.S., et al. (1997). Moderate-intensity exercise and self-rated quality of sleep in older adults: a randomized controlled trial. Journal of the American Medical Association, 277(1), 32-37.

Kripke, D.F., Garfinkel, L., Wingard, D.L., et al. (2002). Mortality associated with sleep duration and insomnia. Archives of general psychiatry, 59(2), 131-136.

Lee, S.H., Han, J.H., Jin, Y.Y., et al. (2016). Poor physical fitness is independently associated with mild cognitive impairment in elderly Koreans. Biology of Sport, 33, 57-62.

Lenz, E. K., Swartz, A. M., \& Strath, S. J. (2014). Do sedentary behaviors modify the health status of older adults? International Journal of Kinesiology \& Sports Science, 2(1), 13-22. 
Li, F., Fisher, K.J., Harmer, P., et al. (2004). Tai Chi and self-rated quality of sleep and daytime sleepiness in older adults: a randomized controlled trial. Journal of the American Geriatrics Society, 52(6), 892-900.

Lindh, M. (1979). Increase of muscle strength from isometric quadriceps exercises at different knee angles. Scandinavian Journal Rehabilitation Medicine, 11, 33-36.

Lira, F. F., Pimentel D.G., Santos R.VT., et al. (2011). Exercise training improves sleep pattern and metabolic profile in elderly people in a time-dependent manner. Health and Disease, 10, 113.

Litchke, L.G., Hodges, J.S., Reardon, R.F. (2012). Benefits of Chair Yoga for Persons with Mild to Severe Alzheimer's Disease. Activities, Adaptation \& Aging, 36(4), 317-328.

Morin, C.M., Mimeault, V., Gagné, A. (1999). Nonpharmacological treatment of late-life insomnia. Journal of psychosomatic research, 46(2), 103-116.

Oken, B.S., Zajdel, D., Kishiyama, S., et al. (2006). Randomized, controlled, six-month trial of yoga in healthy seniors: effects on cognition and quality of life. Alternative therapies in health and medicine, 12(1), 40.

$\mathrm{Pa}$, J., Goodson, W., Bloch, A., et al. (2014). Effect of exercise and cognitive activity in community-dwelling older adults with cognitive complaints: a randomized controlled trial. Journal of American Geriatrics Society, 62, $2319-26$.

Petersen, R.C., Morris J.C. (2005). Mild Cognitive impairment as a clinical entity and treatment target. Archives of Neurology, 62, 1160-1163.

Petersen, R.C., Smith, G.E., Waring, S.C., et al. (1999). Mild cognitive impairment: clinical characterization and outcome. Archives of neurology, 56(3), 303-308.

Ramos-Jimenez, A., Hernandez-Torres, R.P, Wall-Medrano, A., et al. (2009). Cardiovascular and metabolic effects of intensive Hatha Yoga training in middle-aged and older women form northern Mexico. International Journal Yoga, 2, 49-54.

Reid, K.J., Baron, K.G., Lu, B., et al. (2010). Aerobic exercise improves self-reported sleep and quality of life in older adults with insomnia. Sleep medicine, 11(9), 934-940.

Rikli, R.E., Jones, C.J. (2001). Senior fitness test manual. Champaign (IL): Human Kinetics.

Rikli, R.E., Jessie Jones, C. (1999). Development and validation of a functional fitness test for community-residing older adults. Journal of aging and physical activity, 7, 129-161.

Schutte-Rodin, S., Broch, L., Buysse, D., et al. (2008). Clinical guideline for the evaluation and management of chronic insomnia in adults. Journal of clinical sleep medicine: JCSM: official publication of the American Academy of Sleep Medicine, 4(5), 487.

Shakoor, E., Salesi, M., Koushki, M., et al. (2016). The Effect of Concurrent Aerobic and Anaerobic Exercise on Stress, Anxiety, Depressive Symptoms, and Blood Pressure in Renal Transplant Female Patients: A Randomized Control Trial. International Journal of Kinesiology and Sports Science, 4(4), 25-31.

Sharif, F., Sddigh, M., Jahanbin, I., Keshavarzi, S. (2015). The effect of aerobic exercise on quantity and quality of sleep among elderly people referring to health centers of Lar City, Southern of Iran, A randomized controlled clinical trial. Current Aging Science, 8(3), 248-55.

Brockton West Roxbury, V.A (1977). Sleep, Sleep Deprivation, and Daytime Activities A Randomized Controlled Trial of the Effect of Exercise on Sleep. Sleep, 20(2), 95-101.

Tran, M.D., Holly, R.G., Lashbrook, J., Amsterdam, E.A. (2001) Effects of Hatha yoga practice on the health-related aspects of physical fitness. Preventive Cardiology, 4,165-70.

Wilson, N.M., Hilmer, S.N., March, L.M., et al. (2011). Associations between drug burden index and falls in older people in residential aged care. Journal of the American Geriatrics Society, 59(5), 875-880.

Yaffe, K., Laffan, A.M., Harrison, S.L., et al. (2011). Sleep-disordered breathing, hypoxia, and risk of mild cognitive impairment and dementia in older women. Journal of the American Medical Association, 306(6), 613-619.

Yang, K. (2007). A review of yoga programs for four leading risk factors of chronic diseases. Evidenced Based Complement Alternative Medicine, 4, 487-91. 